\title{
An in planta, Agrobacterium-mediated transient gene expression method for inducing gene silencing in rice (Oryza sativa L.) leaves
}

Aurélie Andrieu', Jean Christophe Breitler ${ }^{1}$, Christelle Siré ${ }^{1}$, Donaldo Meynard ${ }^{1}$, Pascal Gantet ${ }^{2}$ and Emmanuel Guiderdoni ${ }^{*}$

\begin{abstract}
Background: Localized introduction and transient expression of T-DNA constructs mediated by agro-infiltration of leaf tissues has been largely used in dicot plants for analyzing the transitivity and the cell-to cell movement of the RNAi signal. In cereals, however, the morphology of the leaf and particularly the structure of the leaf epidermis, prevent infiltration of a bacterial suspension in cells by simple pressure, a method otherwise successful in dicots leaves. This study aimed at establishing a rapid method for the functional analysis of rice genes based on the triggering of RNA interference (RNAi) following Agrobacterium-mediated transient transformation of leaves.

Results: Using an agro-infection protocol combining a wound treatment and a surfactant, we were able to obtain in a reliable manner transient expression of a T-DNA-borne uidA gene in leaf cells of japonica and indica rice cultivars. Using this protocol to transiently inhibit gene expression in leaf cells, we introduced hairpin RNA (hpRNA) T-DNA constructs containing gene specific tags of the phytoene desaturase (OsPDS) and of the SLENDER 1 (OsSLR1) genes previously proven to trigger RNAi of target genes in stable transformants. SiRNA accumulation was observed in the agro-infected leaf area for both constructs indicating successful triggering of the silencing signal. Accumulation of secondary siRNA was observed in both stably and transiently transformed leaf tissues expressing the HpRNA OsSLR1 construct. Gene silencing signalling was investigated in monitoring the parallel time course of OsPDS-derived mRNA and siRNA accumulation in the agro-infiltrated leaf area and adjacent systemic sectors. The sensitive RT-Q-PCR method evidenced a consistent, parallel decrease of OsPDS transcripts in both the agroinfiltred and adjacent tissues, with a time lag for the latter.
\end{abstract}

Conclusions: These results indicate that the method is efficient at inducing gene silencing in the agro-infected leaf area. The transfer of low amounts of siRNA, probably occurring passively through the symplastic pathway from the agro-infected area, seemed sufficient to trigger degradation of target transcripts in the adjacent tissues. This method is therefore well suited to study the cell-to-cell movement of the silencing signal in a monocot plant and further test the functionality of natural and artificial miRNA expression constructs.

Keywords: Agrobacterium tumefaciens, Gene silencing, Leaf agroinfection, OsPDS, OsSRL1, Rice, Transitivity, Cross silencing

\footnotetext{
* Correspondence: emmanuel.guiderdoni@cirad.fr

'CIRAD, UMR AGAP, TAA108/03, Av Agropolis, F-34398 Montpellier Cedex 05,

France

Full list of author information is available at the end of the article
} 


\section{Background}

In dicots plants, agro-infection methods allow efficient transfer and transient expression of T-DNA vectors in leaf cells. Transient assays by agro-infiltration have thus been increasingly used as a simple and rapid method for assaying gene function (Wroblewski et al. 2005; Small 2007). Because they provide a rapid, versatile and convenient way for achieving a very high level of gene expression in a distinct and defined zone of the leaf, these Agrobacterium-mediated transient expression systems have been largely used for analyzing the induction of the RNA silencing process, thereby validating gene function through down regulation of gene expression as illustrated recently in grapevine (Bertazzon et al. 2012)- and analyzing the mechanism of transitivity and movement of RNA silencing in leaves (e.g. Schöb et al. 1997, Voinnet 2001).

In rice, as in other cereals, the establishment of such a system is hindered by the monocot leaf structure which includes the presence of an epidermal cuticular wax and high silicium content preventing infiltration of bacterial suspension by simple pressure. Microprojectile bombardment has been used as an alternative delivery method to introduce expression construct in cereal leaf cells. As to gene silencing, bombardment-mediated introduction of dsRNA corresponding to endogenous genes or transgenes in cells of maize, barley and wheat leaves proved to induce interference with gene function but restricted to the single cell where the delivery is achieved (Schweizer et al. 2000). This stresses the need to establish a rapid, versatile and convenient protocol for achieving a very high level of gene expression in planta e.g. in a distinct and defined zone of the cereal leaf.

Once established and among a range of applications, such a method could be used for triggering a localized, hpRNA-mediated silencing process and further study the transitivity and spreading of the silencing signal throughout the whole plant. RNA silencing is non-cell autonomous and operates through different pathways involving separate mechanisms and, probably, distinct signals (Voinnet 2005a; Brodersen and Voinnet 2006; Ghildiyal and Zamore 2009). The systemic silencing signal reflects with many evidences an antiviral defence mechanism in plants: non-cell autonomous silencing relies on a systemic signal that moves ahead or follows RNA viruses by the same way of propagation to initiate RNA silencing and prevent or delay viral infection (Voinnet 2005b). While RNA-silencing induction and RNA degradation have been studied in detail, much less is known about why and how RNA silencing moves from cell-to-cell and sometimes spreads systemically in plants. The answer to these questions seems to be closely related to the transitive RNA silencing observed in C. elegans and plants. In these organisms, RNA silencing can be amplified by a phenomenon called transitivity which increases the initial pool of siRNAs by producing secondary siRNAs corresponding to sequences located outside the primary targeted regions of a transcript (Nishikura 2001; Sijen et al., 2001). Himber et al. (2003) have proposed a model of RNA silencing cell-to-cell movement including a central role for transitivity. The vast majority of experimental data used to formulate this model of RNA silencing movement over cells and organism was accumulated in $N$. benthamiana and $A$. thaliana. Regarding transitivity of RNA silencing and signalling, experience on monocotyledonous plants remains fairly limited since the report of the conservation of the transitive RNA silencing machinery in rice by Miki et al. (2005).

The goal of this study was first to establish a standardized Agrobacterium transfection protocol for inducing transient gene expression in rice leaf cells. The second objective was to illustrate this method in inducing the silencing of two endogenous rice genes (OsPDS and OsSRL1) to investigate the transitivity and systemic spreading of the RNAi signal in a monocotyledonous leaf.

\section{Methods}

\section{Plant Material and in planta agroinfection}

Three varieties of rice (Oryza sativa L.) were used in this study: (i) the sub tropical japonica rice cv. Zhong Zuo321 from China, (ii) the temperate japonica rice cv. Nipponbare and (iii) the high value indica rice cv. IR64 from IRRI. Bacteria used for rice agroinfection were plated on solid AB medium (Chilton et al. 1974) containing $50 \mathrm{mg} / \mathrm{l} \mathrm{kanamycin}$ sulfate and $75 \mathrm{mg} / \mathrm{l}$ rifampicin and incubated at $28^{\circ} \mathrm{C}$ for 3 days. The bacteria were then collected with a flat spatula and re-suspended in liquid agroinfection medium (R2 Basic (Sallaud et al. 2003) $+10 \mathrm{~g} / \mathrm{l}$ glucose, $200 \mu \mathrm{M}$ acetosyringone, $0,01 \%$ Silwet L-77, pH5.5) by vortexing to give an absorbance at $600 \mathrm{~nm}$ between 0.5 and 0.8 . The antepenultimate emerged leaf of plantlets at tillering stage were mechanically wounded with multiple needles and immersed in the Agrobacterium suspension at $20^{\circ} \mathrm{C}$ for 30 to $60 \mathrm{~min}$. The plantlets were grown for 2 to 3 days at $20^{\circ} \mathrm{C}$ and then placed under containment greenhouse growth conditions. Agrobacterium-mediated transformation of seed embryo-derived embryogenic callus was performed according to Sallaud et al. 2003.

\section{Gene specific tag (GST) and T-DNA constructs}

The binary vector pC5300, a pCAMBIA 1300 derivative, had been described elsewhere (Breitler et al. 2004). The reporter plasmid pC5300-UGN bears the uidA gene under the control of the entire 5' untranslated region of the maize polyubiquitin gene Ubi-1 (Christensen and 
Quail 1996) and the polyadenylation sequence from $A$. tumefaciens nos gene. The hpRNA binary vector pBIOS 738, kindly provided by W. Paul and P. Perez (BIOGEMMA, Clermont-Ferrand, France), was constructed by cloning the intron of rice tubulin 1 gene between twin Gateway $^{\mathrm{TM}}$ cassettes in inverted orientation into a pCAMBIA 2300 binary vector. This hpRNA expression cassette is under the control of the cassava vein mosaic virus (CsVMV) promoter (Verdaguer et al. 1998) and the polyadenylation sequence from $A$. tumefaciens nos gene (Figure 1).

For the rice $P D S$ gene a $400 \mathrm{bp}$ fragment (Accession no. AF049356.1, nucleotides no. 660-1040) was used as GST (forward primer: 5'-GAAGTTTGCTCTTG GACTTTTGCCA-3' and reverse primer: 5'-TATTT GAGTTCCATCGGTAAGTGCA-3').

For the SLR1 gene (Accession no. AB030956), the GST (nucleotide 969-1469) is a DNA fragment of 500 bp (forward primer: 5'-AAGTTCGCCCACTT CACCGCAAATC-3' and reverse primer: 5'-TCGAG GAATGAGCCGGAGTTGTGGT-3'). The hpRNA binary vectors were introduced into Agrobacterium strain EHA105 by electroporation.

\section{RNA analysis}

Total RNA extraction was performed with Tri-Reagent (Sigma) and was used for both high-molecular-weight and small RNA analysis. Total RNA were separated on $17.5 \%$ denaturing polyacrylamid gels and then transferred to nylon membranes (Hybond NX, Amersham). Perfect-Hyb buffer (Sigma) was used for hybridization. Probes were DNA fragments labelled by random priming incorporation of $[32 \mathrm{P}] \mathrm{dCTP}$
(Amersham). The GST probe was PCR products similar to GST cloned in the pBIOS738 binary vector. The 5 ' probes were produced by PCR using the following set of primers: 5'pds, 5'-GGTGCTTCGCAAGTAG CAGC-3' and 5'-TACTAAGAAACAATGAAATG-3' and 5'slr1, 5'-GTGCAAGGACAAGGTGATGG-3' and 5'GCACGCCCACTTCTACGAGT-3'. The 3' probes were produced by PCR with the following set of primers used for RT-PCR analysis 3'-pds, 5'-TTGTGCTCAGTCTG TAGTGGA-3' and 5'-TGTGAAGGGATTAAGAGACC-3' and 3'-slr1, 5'-GGCACAATTGAAGCTTGACG-3' and 5'-ATAGATGGGCTAGGAGGACCAAG-3'. Following hybridisation, the membrane was washed twice for $20 \mathrm{~min}$ at $50^{\circ} \mathrm{C}(2 \mathrm{x} \mathrm{SSC}$ and $2 \% \mathrm{SDS})$ and all hybridisation signals were detected by phosphorimaging (Storm 820, Amersham).

\section{Real-time PCR analysis}

Total RNA extraction was performed with Tri-Reagent (Sigma) according to the manufacturer's instructions. The template DNA was removed by treatment with the DNA RQ1 RNase-free DNase kit (Promega). Each cDNA sample was subjected to real-time PCR analysis in triplicate. To normalize the variance among samples, OsExp (Os06g11070.1) was used as endogenous control (Caldana et al. 2007). Relative expression values were calculated after normalizing against the control cDNA. Primers were designed from 3' end of the gene using Primer3plus (http://www.bioinformatics.nl/ cgi-bin/primer3plus/primer3plus.cgi) with QPCR parameters 'on'. Each primer pair was checked for their specificity using the Primer Blaster tool in OryGenesDB (http://orygenesdb.cirad.fr/tools.html), which were further confirmed by dissociation curve analysis obtained after

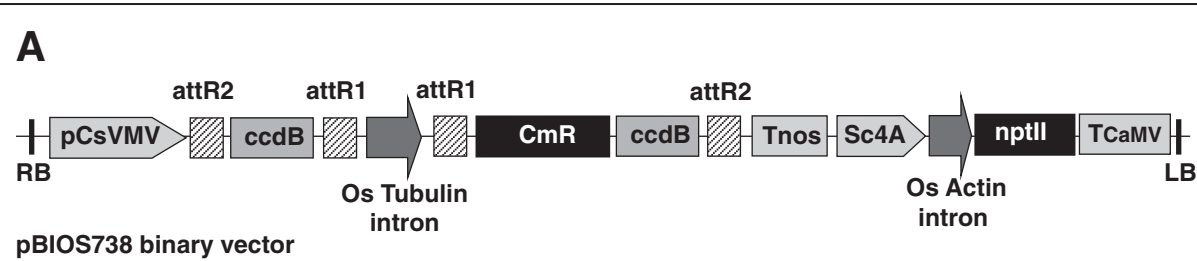

B

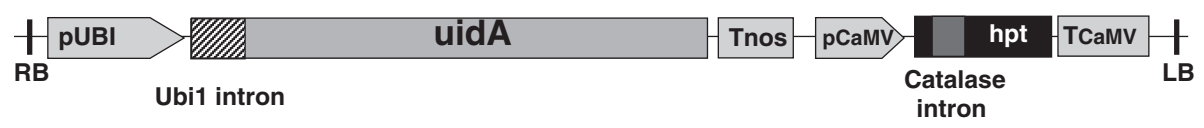

pC5300-UGN binary vector

Figure 1 Schematic maps of the pBIOS738 (W. Paul, Biogemma) and pC5300-UGN T-DNAs. (A) The inverted repeat gateway cassette cloned between the $\mathrm{O}$. sativa tubulin intron is under the control of the strong constitutive promoter of the cassava vein mosaic virus (CsVMV). Sc4A: promoter of the fourth subunit of the subterranean clover mosaic virus. nptll: neomycin phospho transferase II: Tnos : terminator of the Agrobacterium nopaline synthase gene: tCaMV : 3' sequence of the Cauliflower Mosaic Virus (B) The core structure of the pC5300-UGN T-DNA. pUbi: promoter of the maize polyubiquitin 1 gene. uidA: E. coli $\beta$-glucuronidase coding sequence. 
the QPCR reaction. First strand cDNA was synthesized by reverse transcription using $1.5 \mu \mathrm{g}$ of total RNA in $20 \mu \mathrm{l}$ of reaction volume using SuperScriptIII (Invitrogen) as per manufacturer's instructions. Diluted cDNA samples (1/10) were used for Real time PCR analysis with $200 \mathrm{mM}$ of each gene specific primer mixed with SYBR Green PCR master mix in a final volume of $15 \mu \mathrm{l}$ following manufacturer's instructions. The reaction was carried out in 96-well optical reaction plates (Roche), using the Light Cycler 480 Sequence Detection System and software (Roche).

Primer sequences were: 5'-pds, 5'-GTTCCTGATCGAGT GAACGATG-3', 3'-pds, 5'CGAACATGGTCAACAATAGGC3'; 5'OsExp, 5'-CGGTTAGCTAGAGTTCATGTGAGA-3', and 3'OsExp, 5'ATTGGAGTAGTGGAGTGCCAAA-3'.

\section{Results}

\section{Agroinfection of rice leaf tissues with an A. tumefaciens suspension}

To establish an efficient method for Agrobacterium infection of rice leaves, we tested different $A$. tumefaciens strains (GV2200, EHA105, AGL1, LBA4404, and GV3101) carrying the same binary plasmid as well as different infiltration media and temperature, and assessed the efficiency of T-DNA transfer by expression of the carried uidA gene by GUS histochemical assays. The infiltration media and method of Agrobacterium preparation used for Arabidopsis thaliana floral dip or Nicotiana leaf agroinfiltration were completely inefficient on rice leaves whatever temperature or bacterial cell density applied (data not shown). Only the method of bacteria preparation used for genetic transformation of rice embryogenic calli yielded good results, using hyper-virulent strains such as EHA105, AGL1 or LBA4404. Agrobacterium cells were plated on solid AB medium containing antibiotic and incubated at $28^{\circ} \mathrm{C}$ for 3 days. The bacteria were then collected with a flat spatula and diluted in liquid agroinfection medium to optimal bacterial cell density ranging between 0.5 and 0.8 . Because A. tumefaciens was not able to penetrate in the leaf through epidermal cuticle or via sub-stomatal chamber, we built a small apparatus carrying many $600 \mu \mathrm{m}$ diameter needles (Figure 2A) to quickly produce a large number of wounds on the rice leaf surface. To avoid tissue yellowing or wilting, rice leaves were incubated in the Agrobacterium suspension for 30 to 60 minutes (Figure 2B). The first experiments, carried out in the containment greenhouse at $27-28^{\circ} \mathrm{C}$, conducted to very weak uidA transgene expression, upon GUS histochemical assay (data not shown). Consequently, the temperature was reduced to $20^{\circ} \mathrm{C}$ during incubation. Moreover, supplementation of the agro-infection medium with Silwet L-77, a powerful surfactant, proved necessary to reach a high level, transgene expression. A two-fold concentration of acetosyringone $(200 \mu \mathrm{M})$ also increased GUS staining intensity. For all rice genotype tested, high levels of GUS activity were observed 2 to 4 days following soaking in Agrobacterium cell suspensions of EHA105, LBA4404 and AGL1 strains. GUS staining was not detected any more after 10 days (Figure 2C). No GUS staining was observed in unwounded and soaked leaves and in wounded but unsoaked leaves (not shown). This protocol was also used successfully for transient expression of the uidA gene in the roots of rice plants grown under hydroponics (data not shown).

\section{Analysis of RNAi in HpRNA T-DNA stable transformants}

To determine whether RNA silencing can be initiated in agro-infected rice leaves by our method, we selected two endogenous gene targets namely Phytoene Desaturase (OsPDS) and SLENDER 1 (OSSLR1 or OsGAI, a rice ortholog of the height-regulating gene GAI/RGA/RHT/ D8) because of their well-documented loss-of function phenotype. $P D S$ has been used extensively to investigate gene silencing both in dicots (Voinnet 2001) and monocots (Miki et al. 2005). We first analyzed the endogenous mRNA level present in different tissues of wild type Nipponbare plants. We carried out northern blot analyses using the sequences of OsPDS and OsSLR1 Gene Specific Tags (GST) as probes to hybridize sequentially the same membranes. In callus and leaves of six day-old seedlings, transcript accumulation of OSPDS and OsSLR1 were rather similar. Contrastingly, in leaves of 5-6 leaf stage plantlets, OsPDS mRNAs accumulated at a higher level than those of OsSLR1 (Figure 3A). To determine the efficacy of the hpRNA construct in triggering RNA silencing and thereby conducting to the anticipated loss of function phenotype, stable transgenic rice lines were produced through co-culture of 25 embryogenic calli of cv Nipponbare. Analysis of 85 OsPDS RNAi T0 lines plants showed various levels of $P D S$ mRNA degradation, leading to phenotypes ranging from wild type to fully albino plants, in which the PDS gene was found to be strongly silenced (Figure 3B). Primary 21-nts-siRNAs, a molecular marker for dsRNA-based gene silencing, were easily detected in all albino lines which accounted for $54 \%$ of the regenerated plants (Figure 3C). A correlation was observed between the accumulation of siRNA and severity of the phenotype. Though a similar number of rice embryogenic calli was co-cultivated with Agrobacterium bearing the OsSLR1 hpRNA construct, only four plants regenerated from transformed cell lines displayed a strong mutant phenotype but which sharply contrasted with that of slr1 (Figure 3B) while other regenerated lines displayed a normal WT phenotype. Mutant plants were found to accumulate siRNAs whereas WT regenerants did not (Figure 3C). Based on the phenotype 

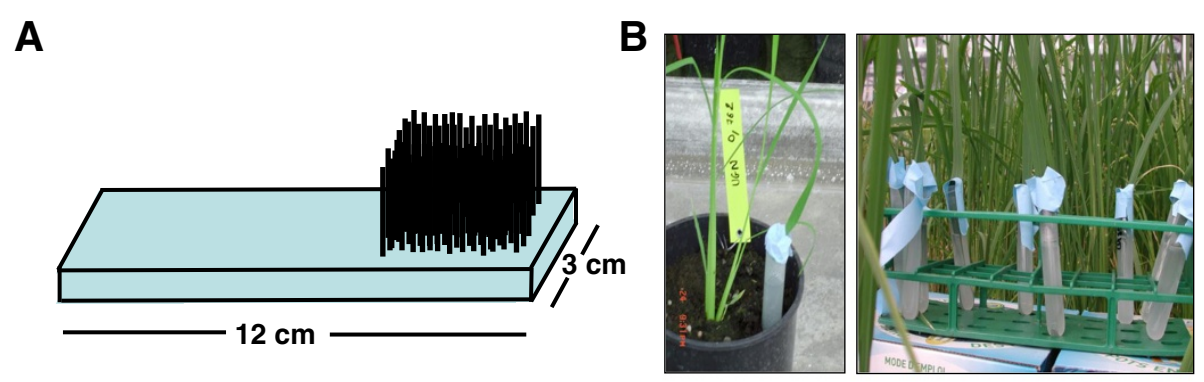

C

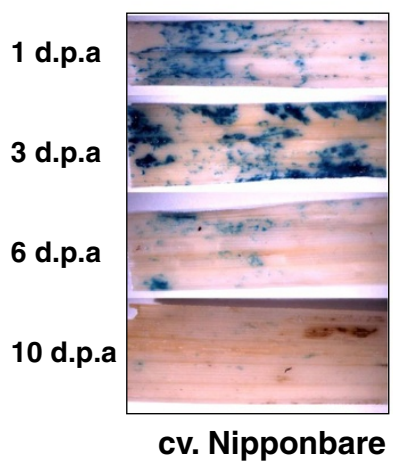

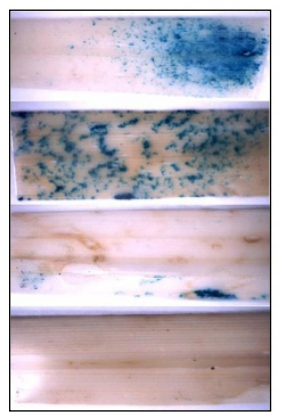

cv. Zhong Zuo 321

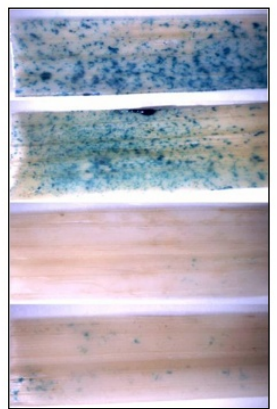

CV. IR64

Figure 2 Agroinfection of rice leaf tissues with $\boldsymbol{A}$. tumefaciens suspension. Histochemical assays to assess the expression of the uidA gene in rice tissues were carried out by staining with 5-bromo-4-chloro-3-indolyl-beta-D-glucuronid acid as described by Jefferson (1987). (A) Schematic representation of the small apparatus carrying many needles used to wound rice leaves. (B) Wounded rice leaves soaking in the Agrobacterium suspension in containment greenhouse. (C) GUS staining observed after 1, 3, 6 and 10 days post agroinfection (d.p.a) for three different cultivars of rice. No GUS staining was observed in unwounded and soaked leaves and in wounded but unsoaked leaves (not shown).

description of slr1 mutants by Ikeda et al. (2001) we were indeed expecting a constitutive gibberellin response that leads to a tall phenotype. Though the OSSLR1 is unique in the rice genome, the sequences of four other gene members of the GRAS family (namely Os01g45860.1 also called SLRL1, Os05g49990.1, Os11g03110.1 and Os12g06540.1) (Additional file 1: Figure S1) share high similarity with a putative $21 n t-s i R N A$ residing in the OsSLR1 GST sequence (Additional file 2: Figure S2-A). This makes them candidates prone to targeting by OsSLR1 primary siRNA that accumulate in the HpRNAi slr1 mutants (Additional file 2: Figure S2-B) through a mechanism called cross-silencing. Hybridization of small RNA blots of the HpRNAi slr1 mutant with probes specific to each of these 4 genes evidenced accumulation of 21 nt siRNAs for 3 of them (Additional file 2: Figure S2-C). It is therefore likely that this cross silencing accounts for the contrasting phenotype observed in our HpRNAi slr1 mutant with regards to that of the reference disruption mutant.

\section{Analysis of the RNA silencing in leaves following agroinfection}

To determine whether agro-infection of leaf cells conducts to local induction of RNA silencing following transcription of the hpRNA construct, we agroinfected leaves of wild type Nipponbare plants using the T-DNA construct carrying the OSPDS and OsSLR1 hpRNA structures. pBIOS738 empty vector was used as control over the time course experiment. RNA was isolated from the agro-infected regions of the leaf and analysed for the accumulation of transcripts and of siRNAs, 2, 4, 8, 12 and 20 days following inoculation (Figure 4A and B). Each time point involved at least three biological replicates. In the agro-infected region of the leaf, OSPDS and OSSLR1 siRNAs were consistently detected from day 2 post-agroinfection. The image analysis software, ImageQuant ${ }^{\mathrm{TM}}$ TL (Amersham), was used to quantify siRNAs detected by northern blotting (Figure $4 \mathrm{C}$ ). The OsPDS siRNAs concentration peaked at day 12 and dramatically decreased to reach a very low level at day 20, in the two first experiments. On the other hand, we were able to detect OsPDS siRNA 30 days after agro-infection in a third experiment (data not shown). Accumulation of the OsSLR1 siRNAs was still occurring at day 20 though likely reaching a plateau. In each experiment, a population of plants agro-infected was further grown in the containment greenhouse and phenotypic observations were regularly performed. These plants displayed the 


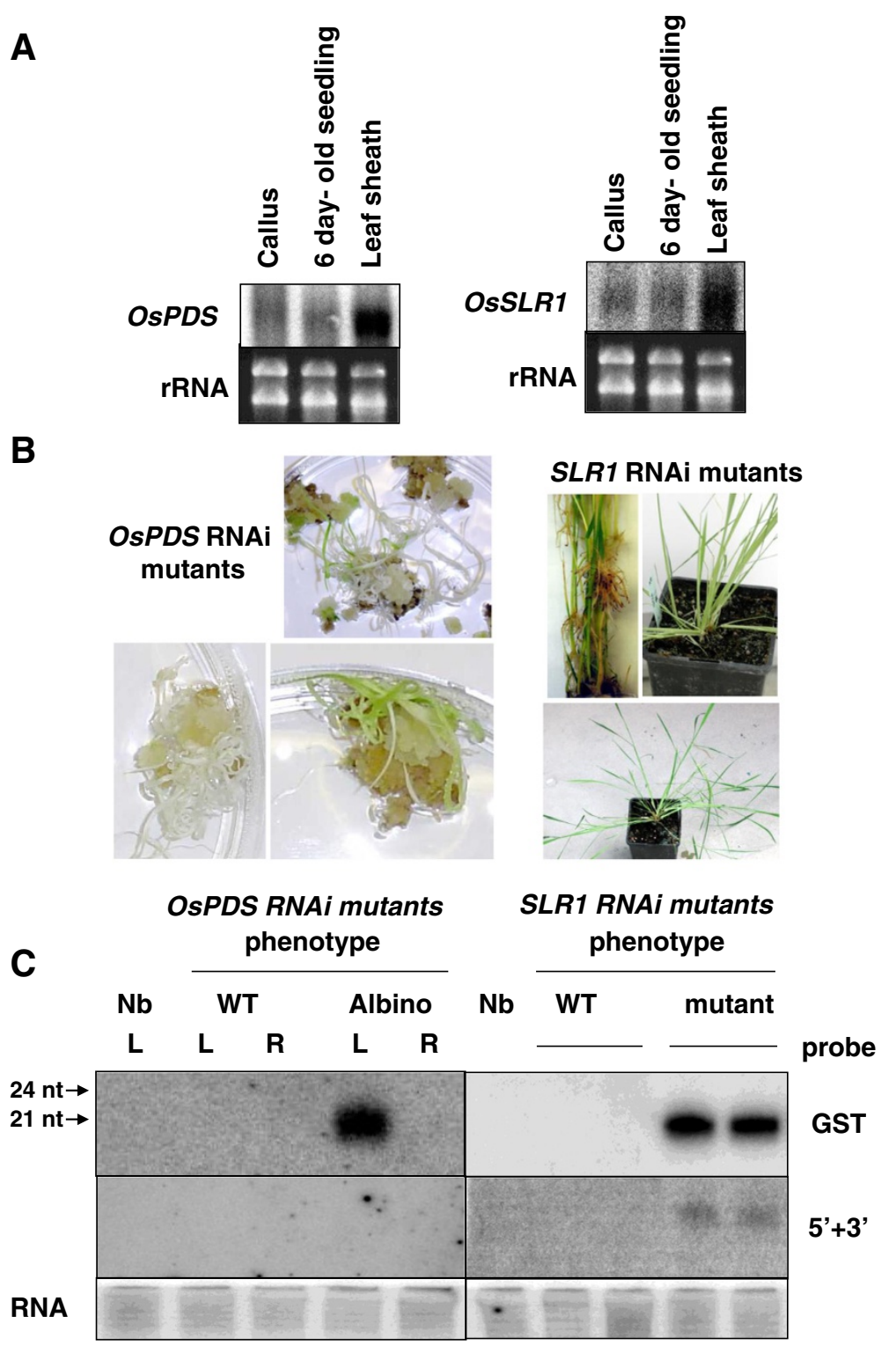

Figure 3 Analysis of HpRNA T-DNA stable transformants. (A) Northern blot detection of OSPDS and OsSLR1 mRNA accumulation in different tissues of wild type plants of cv. Nipponbare. (B) Phenotypes of RNAi mutant lines expressing OsPDS RNAi HpRNA construct. (C) Detection of 21-nts siRNAs in OSPDS and OsSLR1 HpRNA transgenic lines exhibiting or not a mutant phenotype. A probe specific to the gene specific tag (GST) and a probe representing regions extending $3^{\prime}$ and $5^{\prime}$ from the GST interval have been sequentially used. L (leaf), R (root), Nb (Control Nipponbare plant).

same phenotype as the control plants throughout their life cycle. No siRNA could be detected in the tissues of non agro-infected leaves of higher and lower rank, whatever the hpRNA construct used, suggesting that very low or null long distance siRNA movement occurred through the phloem. In order to test whether the pattern of siRNA formation could be reproduced in indica rice, we repeated the agro-infection experiment with the same construct in leaves of $\mathrm{cv}$. IR64. The siRNA accumulation pattern, analysed over 30 days post agro-infection, was fully consistent with that previously observed in the japonica cv. Nipponbare. Throughout the experiment and in both cultigens of rice, siRNA were not detected in the non agro-infected leaves.

\section{OsPDS and OsSRL1 silencing transitivity in rice leaf} tissues

In order to determine whether a transitive RNAi mechanism has been initiated in mutant lines and in agroinfected rice leaves, we hybridized the available blots with probes corresponding to regions the extending 5' and 3' from the trigger dsRNA interval in the two 


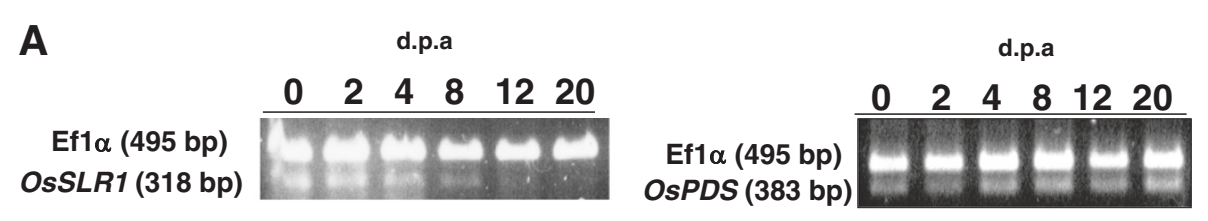

B
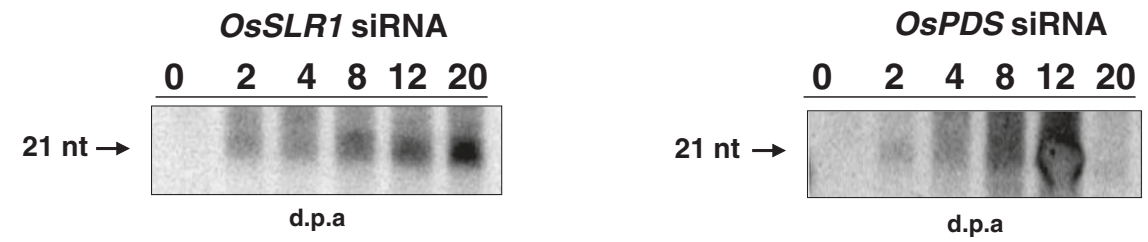

C
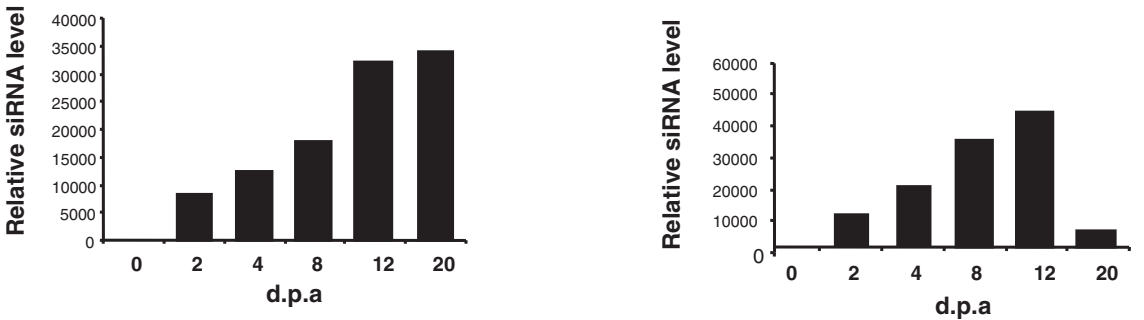

Figure 4 Analysis of the agroinfiltrated leaf tissues. (A) Accumulation of OSPDS and OSSLR1 mRNAs revealed by RT-PCR during the time course experiment. Ef1a mRNA was used as a standard. (B) Time course of OsPDS and OsSLR1 siRNAs accumulation following leaf agroinfection. Detection of siRNAs was performed using a GST probe. Arrow indicates the 21-nt-size-class siRNAs. Fifteen $\mu \mathrm{g}$ of total RNA extracted from leaf tissues after 2, 4, 8, 12 and 20 days were loaded on gel. A parallel time course was achieved with leaf tissues of plants agro-infiltrated with the pBIOS738 empty vector that served as reference value for siRNA accumulation. (C) Quantification of siRNAs detected by northern blotting using the ImageQuant ${ }^{\mathrm{TM}} \mathrm{TL}$ image analysis software.

genes. In both agro-infected leaf areas and in stably transformed leaf tissues expressing the OsPDS hpRNA construct, siRNAs corresponding to the region used to produce the trigger dsRNA were detected but no siRNA was detected when the regions extending either 5' or 3' from the trigger dsRNA borders were used as probe (Figure 3C). The membranes were re-hybridized with success using the trigger dsRNA probe to ascertain the presence of siRNAs on the filters following repeated cycles of hydridization-dehybridization. Contrastingly, siRNA corresponding to regions extending $5^{\prime}$ and 3 ' from the trigger RNA interval accumulated in stably and transiently transformed leaf tissues expressing the OsSLR1 hpRNA construct (Figures $3 \mathrm{C}$ and $5 \mathrm{~A}$ and $\mathrm{B}$ ). As the probes used were long the possibility of nonspecific cross reaction between the 5' and 3' probes and the siRNAs derived from the silencing triggering hpRNA could not be ruled out. In order to ascertain hybridization specificity, the Ambion online siRNA target finder was used to identify all the potential siRNA from 5', GST and 3' part of OsSLR1 coding sequence. Thirteen, 12, and 5 potential siRNAs were identified in these 3 respective regions (Figure $5 \mathrm{C}$ ). It is assumed that nearly $50 \%$ of the siRNAs designed using this tool will achieve a $>50 \%$ reduction of target gene expression. Considering this, oligo-nucleotides corresponding to each group were mixed in equimolar ratio and used as probe to re-hybridize small RNAs from leaves expressing the OsSLR1 HpRNA construct (Figure 5D). Despite detection of lower signals, these experiments confirmed results obtained using longer probes. As a glimpse of Ambion software efficiency, potential siRNAs detected along the OsSLR1 GST sequence were sequentially used as probe to re-hybridize RNAs extracted from $\operatorname{slr} 1 \mathrm{mu}$ tant lines. Five $(41.6 \%)$ of 12 potential siRNAs showed a clear hybridization signal (data not shown).

\section{Variation of OsPDS transcript accumulation along the agroinfected leaf}

To investigate the spreading of the RNAi silencing signal along the rice leaf, we compared the mRNA level of the target gene in agro-infected and adjacent leaf tissues by quantitative RT-PCR (Figure 6A). As the HpRNA construct-mediated silencing of the OSSLR1 gene was prone to a complex cross silencing phenomenon that might be specific to the gene, we focused this experiment on tissues infiltrated with the OsPDS HpRNA construct. For q-RT-PCR, the gene of interest is co-amplified with an 


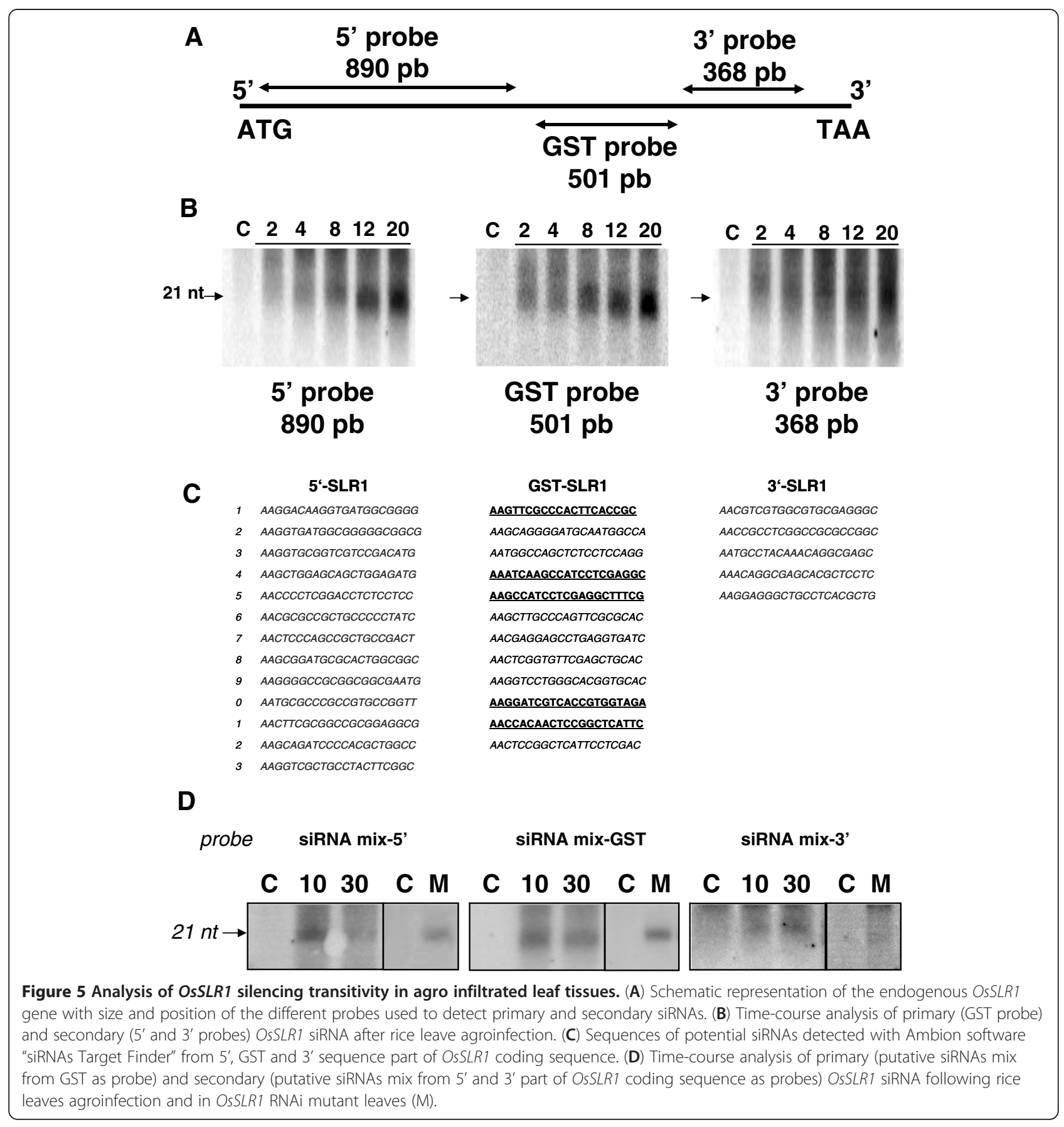

internal control gene to determine the relative abundance of target gene transcripts in the leaf tissue. OsExp, similar to its Arabidopsis ortholog (At4g33380), was chosen as the internal control because this gene showed good stability and its amplification remains in a log-linear stage at the same optimal conditions as those of target genes. Analysis of leaves agroinfected with the OsPDS hpRNA construct showed a close relationship between hpRNA transient expression and depression of PDS transcripts level. Compared to the wild type, the quantity of OsPDS mRNA fell to $29 \%$ two days after the agroinfection, and then reached a minimum of $10 \%$ at day 4 . mRNA accumulation in agroinfected tissues reached $17 \%$ then $44 \%$ of the wild type value at day 8 and 12 respectively (Figure 6B). Similar results were observed for adjacent tissues with those agroinfected with a 4 day-shift for the peak of degradation of OsPDS mRNA which reaches $10 \%$ of the value of the wild type 8 days following agro-infection. 

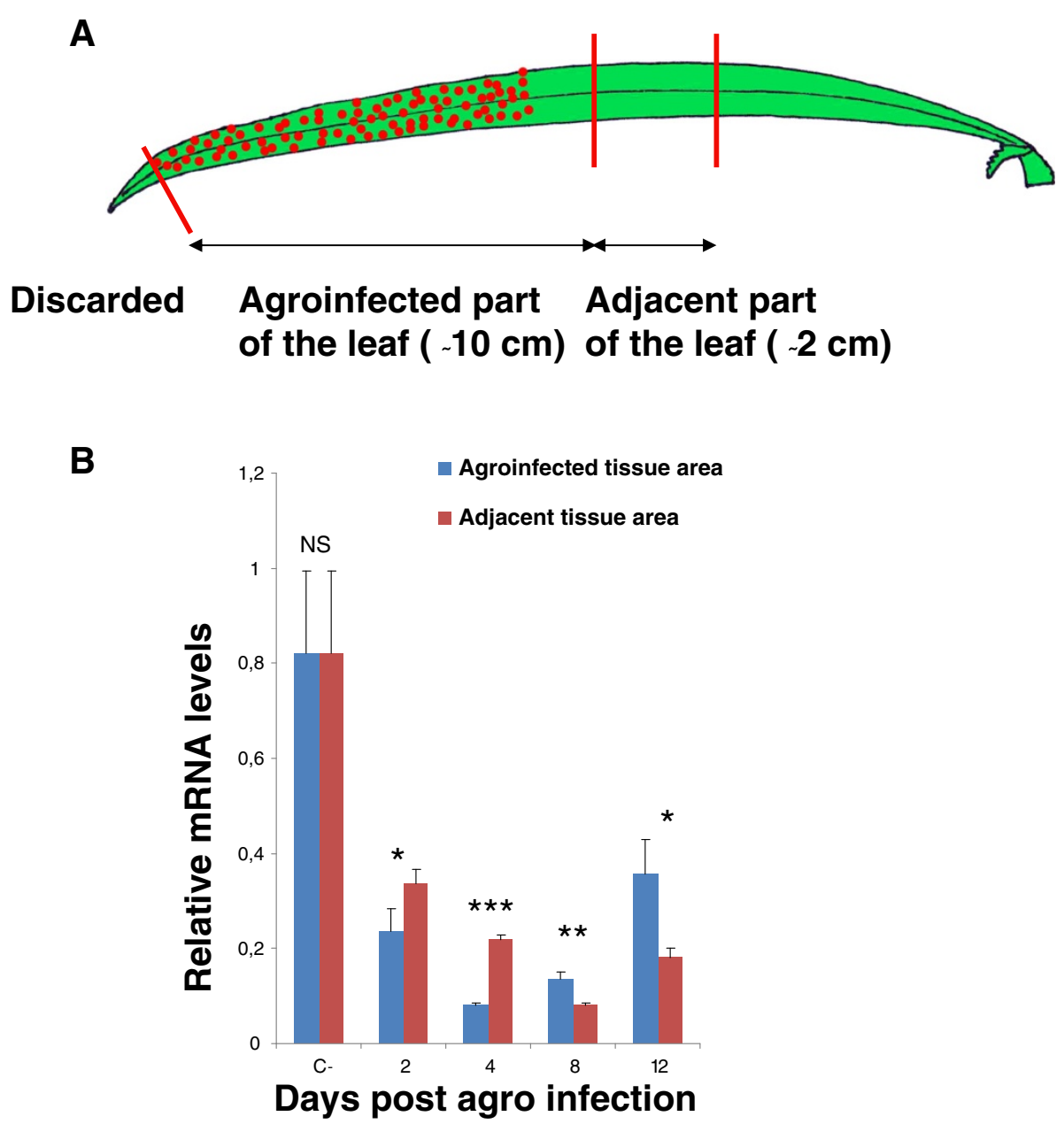

Figure 6 Analysis of the movement of the OsPDS silencing signal from agroinfiltrated leaf tissues. (A) Schematic representation of the rice leaf. Wounded and agroinfected part of the leaf and adjacent part are indicated. (B) Real-time PCR time course analysis of OsPDS transcript accumulation in the agro-infiltrated and adjacent region of the leaf. Results of 3 biological replicates are shown. Primer sequences were: $5^{\prime}-p d s$, 5'-GTTCCTGATCGAGTGAACGATG-3', 3'-pds, 5'-CGAACATGGTCAACAATAGGC-3'; 5'-OsExp, 5'-CGGTTAGCTAGAGTTCATGTGAGA-3', and 3'-OsExp, $5^{\prime}$-ATTGGAGTAGTGGAGTGCCAAA-3'. NS, ${ }^{*},{ }^{* *}$, and ${ }^{* * *}$ means non significantly different and statistically different at the $5 \%, 1 \%$ and $0.1 \%$ level in a t-student test respectively.

\section{Discussion}

An Agrobacterium-mediated transient expression system for cereal leaves

Because they provide a rapid, versatile and convenient way for achieving a very high level of gene expression in a distinct and defined zone of leaf, Agrobacterium-mediated transient expression systems have been chosen for inducing RNA silencing processes and transitivity analysis in leaves (Schöb et al. 1997). The first objective of this study was to evaluate the feasibility of an agro-infiltration approach for transient expression of a T-DNA construct in leaf tissues of rice. In cereal species, the leaf structure is known to prevent infiltration of a bacterial solution by simple pressure. In order to provide an access of Agrobacterium to the leaf cells, we used a combination of multiple uniform wound treatment and supplementation of the suspension with a powerful surfactant. After testing different A. tumefaciens strains as well as different infiltration media and temperatures, assessed by GUS histochemical assays, we have developed an efficient and reproducible method for agroinfection of leaves in indica and japonica rice, based on soaking wounded tissues in EHA105 bacterial solution at low temperature. The most critical physiological parameter appeared to be the post agro-infiltration incubation temperature. The largest and most intensively stained GUS areas in leaves were observed when rice plants are transferred at $20^{\circ} \mathrm{C}$ following agro-infiltration. A hypothesis is that a $25^{\circ} \mathrm{C} / 28^{\circ} \mathrm{C}$ temperature favors bacterial multiplication $v s$. their attachment to plant cells and T-DNA transfer whereas at $20^{\circ} \mathrm{C}$, bacterial multiplication slows down, thereby favoring plant cell-bacteria interactions. As reported in the literature in other plants (Batra and 
Kumar 2003; Wydro et al. 2006), we showed that acetosyringone was essential for efficient transfection, and the use of a two-fold concentration compared to our rice stable transformation procedure increases the efficiency of in planta agroinfection. As reported in other plants species (Wroblewski et al. 2005; Chabaud et al. 2003), the choice of the Agrobacterium strain appeared critical because of specific host-bacteria interactions (Tzfira and Citovsky 2002). In Agrobacterium-mediated in planta transformation, the genotype is generally found to influence T-DNA transfer and transgene expression. This was observed for instance in Arabidopsis and grapevine (Wroblewski et al. 2005; Zottini et al. 2008; Santos-Rosa et al. 2008). However, in our study, all the rice genotypes tested were found to be amenable to Agrobacteriummediated transient expression of the uidA gene in infiltrated leaf cells. The optimized rice agroinfection protocol was also applied with success to sugarcane suggesting that other graminaceous species are amenable to this procedure (unpublished results). This method could be also very useful for transgenic complementation (Van der Hoorn et al. 2000; Shao et al. 2003; Bertazzon et al. 2012), promoter analysis (Yang et al. 2000), protein production (Vaquero et al. 1999) and ascertaining miRNA and amiRNA processing and target cleavage (Warthmann et al. 2008) in rice plants. The method is also applicable at different developmental stages as suggested by similar intensities of GUS staining observed in leaves of plants from the 5 leaf stage to the flowering stage. Our results indeed pointed that the developmental stage of the rice plants is not a critical parameter for an efficient expression of uidA gene induced in agroinfected leaves, though the efficiency becomes lower in old leaves than in seedling leaves probably due to plant resistance to agro-infection acquired at maturity (Wroblewski et al. 2005; Wydro et al. 2006; Santos-Rosa et al. 2008).

\section{HpRNA constructs triggers a maintained RNAi process in agro-infiltrated leaf tissues}

In the experiments described here, we have analysed RNA silencing of two endogenous rice genes in both agro-infected leaf tissues and stably transformed rice plants, constitutively expressing an hpRNA construct. In both situations, we were able to detect siRNAs corresponding to the trigger dsRNA. In stable transgenic lines, the accumulation of which correlated the penetrance of the RNAi mutant phenotype, demonstrating the efficiency of our hpRNA construct in initiating RNA silencing of the corresponding endogenous gene in rice cells. Transient expression of hpRNA after leaf agro-infection was sufficient to trigger RNA silencing. During 10 to 20 days following rice agro-infection depending on the gene targeted, the quantity of siRNAs increased in cells then remains stable or decreased till leaf senescence. Considering that transient expression of the T-DNA-borne genes occurs only during the very few days following agroinfection (as attested by the revelation by histochemical assay of the otherwise rather stable GUS protein, that dramatically decreased between 6 to 10 days after agroinfection (data not shown)), we concluded that RNA silencing was maintained in rice cells in the absence of the trigger hpRNA. Local initiation of silencing produced preferentially primary $21-n t$ siRNA and maintenance of siRNAs production during cell life indicated that target genes are actively transcribed. This result is consistent with previously published data. 21-nt siRNAs are believed to guide mRNA cleavage, whereas 24nt siRNAs are believed to exclusively mediate chromatin modification and transcriptional silencing by acting in a RISC-like complex (Brodersen and Voinnet 2006).

Real-time PCR analysis showed the highest OsPDS transcript depletion in the agro-infected leaf area 4 days following agro-infiltration. These results are consistent with the literature which reports a transitory peak of transgene expression at day 4 following agro-infection in $N$. Benthamiana and A. thaliana. Decrease of OsPDS mRNA degradation parallels the decline of transient expression of the HpRNA construct. Longer term maintenance of the silencing of the OsPDS gene and exhibition of the photobleaching phenotype would have probably required higher siRNA accumulation in agro infiltrated tissues. High level siRNA could be obtained in enhancing Agrobacterium-mediated delivery in leaf cells or through an amplification of the production of siRNA resulting from transitivity mechanism (see following section). Using the same hpRNA construct bearing the OsPDS GST sequence to initiate RNAi in tobacco leaves following agro-infection, we observed a similar amplification of the siRNAs and their maintenance in tissues where the initiation took place, but resulting in a photobleaching phenotype (unpublished results). The most obvious difference between rice and tobacco, at the experimental level, resides in the higher penetration of agrobacteria in leaves and consequently of bacterial cells in contact with leaf cells. In order to obtain an amplification of the degradation of the mRNA and to observe a phenotype in rice leaves, it might be necessary to increase the level of agro-infection in rice tissues.

\section{HpRNA-mediated induction of OsPDS and OsSLR1 silencing differs by the occurrence of transitivity in both stably and transiently transformed leaf tissues}

We were not able to evidence transitivity in both transiently and stably silenced leaf tissues expressing the OsPDS HpRNA construct. This result is consistent with previous reports in tobacco (rubisco and pds genes; Vaistij et al. 2002), Arabidopsis (rubisco and sulfur genes; Himber et al. 2003) and rice (OsGen-L, Moritoh et al. 2005; Ospds and OsRAC genes, Miki et al. 2005). Surprisingly, HpRNA-mediated silencing of the OsSLR1 
gene clearly involved transitivity with formation of siRNA occurring from regions of the endogenous target mRNA extending 5' and 3' from the region containing the trigger siRNA. One possible explanation for apparent lack of transitive RNA silencing of OsPDS endogenous gene may lie in a low concentration of the RNA substrates for RDR (Garcia-Perez et al. 2004). However, northern blot analysis of total RNA extracted from wild type plants clearly showed that transcription level of OsPDS is rather equivalent to that of OSSLR1 in seedling leaves and is even higher at a later developmental stage. Along the same line, silencing of the most abundant mRNA in plants, that of the rubisco small subunit, does not involves transitivity nor long range spreading of the silencing signal (Vaistij et al. 2002; Himber et al. 2003) suggesting that the mRNA level might not be an essential factor in transitive RNA silencing. Experience on the RNA silencing transitivity in monocotyledonous plants is limited to the pioneering report of Miki et al. (2005) whose showed that the transitive RNA silencing machinery is conserved in rice. In this study, whereas transitive RNA silencing occurred in both 5' and 3' orientations for the exogenous $g f p$ gene, no transitivity was observed for the endogenous OsPDS and OsRAC genes. Therefore, the cause of transitivity of the RNAi signal in OsSLR1 remains to be further investigated.

\section{Spreading of the silencing signal to the non-agro infiltrated leaf tissues}

Though the current model of cell-to-cell movement of the RNA silencing signal implies a central role for transitivity (Himber et al. 2003), we surprisingly detected an efficient decrease of the OsPDS mRNA level in the leaf tissue adjacent to the agroinfiltrated area occuring with a time lag. A possible explanation lies in the transfer of the siRNA from agro-infected tissues to healthy tissues through the symplastic pathway as described by Dunoyer et al. (2010). The vast majority of experimental data used to formulate the current model of cell-to-cell movement of RNA silencing signal was accumulated in $N$. benthamiana and A. thaliana. The short range movement (up to 10-15 cells) of the silencing signal requires accumulation of $21 \mathrm{nt}$ but not of $24 \mathrm{nt}$ siRNA while the possibility that ssRNA move from one cell to the other cannot be yet ruled out. The longer range (beyond 10-15 cells) systemic signal appears to be RDR6 dependent since no spreading and no secondary siRNA is observed in $r d r 6$ background. However, that long range spreading has so far been only observed in the RNAi of exogenous transgenes that generally implies extensive production of secondary siRNA and may result in exogenous DNA methylation. SiRNA cell-to-cell movements occur either in a non selective or selective manner through plasmodesmata (Hyun et al. 2011). It is thought that a molecular link exists between RNAimediated DNA methylation and regulation of selective siRNA movement through plasmodesmata but that link remains to be elucidated.

\section{Cross silencing occurred in HpRNA silencing of OsSLR1}

As demonstrated by Miki et al. (2005) with the OsRAC gene family, a single IR of a specific region shared by several genes of a same family can simultaneously suppress the expression of multiple genes. In this work we studied the possibility of cross extinction of rice genes sharing sequence homology outside the sequence of OsSLR1 GST. The results showed that transitivity could be responsible of multiple endogenous genes knock-down. So the choice of a highly conserved and specific sequence, is necessary to precisely suppress only one member in a gene family.

\section{Conclusion}

In conclusion, this work demonstrates that in planta, Agrobacterium-mediated transient expression of T-DNA constructs is achievable in rice leaves. The possibility to trigger RNAi in a localized manner in the rice leaf opens new perspectives to study the cell-to-cell movement of the silencing signal in a monocot plant. This method could also be of particular interest to test the functionality of natural and artificial miRNA expression construct, in ascertaining miRNA processing and cleavage of target genes that are expressed in rice leaves. It is indeed often necessary to assay several amiRNAs, the sequences of which are based on computational predictions, to find one which effectively triggers knock down of a target gene (Warthmann et al. 2008). As this transfection method has been used to achieve localized GUS expression in roots of rice plants grown in hydroponics (data not shown) it should be as well applicable for target genes expressed in other rice organs.

Aurélie Andrieu and Jean Christophe Breitler are first co authors.

\section{Additional files}

Additional file 1: Figure S1. Phylogenetic tree of the members of the Arabidopsis thaliana and Oryza sativa GRAS transcription factor families generated through the GreenphyIDB GOST tool (http://greenphyl.cirad.fr/ V2/cgi-bin/index.cgi). Position of the four GRAS genes exhibiting a shared putative siRNA with OsSLR1 are highlighted.

Additional file 2: Figure S2. (A) Alignments of the shared putative 21 nt-siRNA residing in the sequence of OSSLR1and in those of the four other members of the GRAS family. (B) Detection of the common

putative 21nt-siRNA in transformation events harbouring the OSSLR1 HpRNA T-DNA construct and exhibiting (mutant) or not (wt) a

phenotype. Nb: a control Nipponbare plant. (C) Detection of siRNAs specific to four other members of the GRAS family the sequence of which contains the putative, shared $21 \mathrm{nt}$-siRNA in transformation events 
harbouring the OsSLR1 HpRNA T-DNA construct and exhibiting (mutant) or not (wt) a phenotype. Nb: Control Nipponbare plant.

\section{Competing interests}

The authors declare they have no competing interest.

\section{Authors' contributions}

AA carried out the work of agroinflitration and molecular analyses and co-wrote the manuscript. JCB initiated the research, carried out the work of agroinflitration and molecular analyses with AA and co-wrote the manuscript. CS assisted JCB in siRNA blotting. DM produced stable transformants of OsPDS and OSSLR1. PG supervised the work and edited the manuscript. EG supervised the work and co-wrote the manuscript. All authors read and approved the final manuscript.

\section{Acknowledgments}

We thank the Région Languedoc Roussillon for providing AA with a PhD grant. We thank Dr W. Paul and Dr P. Perez for the pBIOS 738 plasmid and AGRIDYNE S.A. company for providing us with Silwet L-77 surfactant. We thank Dr C. Périn, CIRAD, for valuable advices in the course of this research and assistance in Q-RT-PCR analyses.

\section{Author details}

'CIRAD, UMR AGAP, TAA108/03, Av Agropolis, F-34398 Montpellier Cedex 05, France. 'Université Montpellier II, UMR DIADE, F-34398 Montpellier Cedex 05, France.

\section{Received: 25 November 2011 Accepted: 12 July 2012}

Published: 31 August 2012

\section{References}

Batra S, Kumar S (2003) Agrobacterium-mediated transient GUS gene expression in buffel grass (Cenchrus ciliaris L.). J Appl Genet 44:449-458

Bertazzon N, Raiola A, Castiglioni C, Gardiman M, Angelini E, Borgo M, Ferrari S (2012) Transient silencing of the grapevine gene VVPGIP1 by agroinfiltration with a construct for RNA interference. Plant Cell Rep 31:133-143

Breitler JC, Meynard D, Van Boxtel J, Royer M, Bonnot F, Cambillau L, Guiderdoni E (2004) A novel 2 T-DNA binary vector allows efficient generation of markerfree transgenic plants in three elite cultivars of rice (Oryza sativa L.). Transgenic Res 13:271-287

Brodersen P, Voinnet O (2006) The diversity of RNA silencing pathways in plants. Trends Genet 22:268-280

Caldana C, Scheible W-R, Mueller-Roeber B, Ruzicic S (2007) A quantitative RT-PCR platform for high-throughput expression profiling of 2,500 rice transcription factors. Plant Methods 3:7-16

Chabaud M, De Carvalho-Niebel F, Barker DG (2003) Efficient transformation of Medicago truncatula cv. Jemlong using the hypervirulent Agrobacterium tumefaciens strain AGL1. Plant Cell Rep 22:46-51

Chilton M, Currier T, Farrand S, Bendich A, Gordon M, Nester E (1974) Agrobacterium tumefaciens DNA and PS8 bacteriophage DNA not detected in crown gall tumors. Proc Nat Acad Sci USA 71:3672-3676

Christensen A, Quail P (1996) Ubiquitin promoter-based vectors for high level expression of selectable and/or screenable marker genes in monocotyledonous plants. Transgenic Res 5:213-218

Dunoyer P, Schott G, Himber C, Meyer D, Takeda A, Carrington JC, Voinnet O (2010) Small RNA duplexes function as mobile silencing signals between plant cells. Science 328:912

Garcia-Perez R, Van Houdt H, Depicker A (2004) Spreading of post-transcriptional gene silencing along the target gene promotes systemic silencing. Plant $J$ 38:594-602

Ghildiyal M, Zamore PD (2009) Small silencing RNAs an expanding universe. Nat Rev Genet 10(2):94-108

Himber C, Dunoyer P, Moissiard G, Ritzenthaler C, Voinnet O (2003) Transitivitydependent and -independent cell-to-cell movement of RNA silencing. EMBO J 22:4523-4533

Hyun KT, Uddin MN, Rim Y, Kim JY (2011) Cell-to-cell trafficking of RNA and RNA silencing through plasmodesmata. Protoplasma 248:101-116

Ikeda A, Ueguchi-Tanaka M, Sonoda Y, Kitano H, Koshioka M, Futsuhara Y, Matsuoka M, Yamaguchi J (2001) Slender rice, a constitutive gibberellin response mutant, is caused by a null mutation of the SLR1 gene, an ortholog of the height-regulating gene GAI/RGA/RHT/D8. Plant Cell 13(5):999-1010

Jefferson R (1987) Assaying chimeric genes in plants: The GUS gene fusion system. Plant Mol Biol Rep 5:387-405

Miki D, Itoh R, Shimamoto K (2005) RNA silencing of single and multiple members in a gene family of rice. Plant Physiol 138:1903-1913

Moritoh S, Miki D, Akiyama M, Kawahara M, Izawa T, Maki H, Shimamoto K (2005) RNAi-mediated silencing of OsGEN-L (OsGEN-like), a new member of the RAD2/XPG nuclease family, causes male sterility by defect of microspore development in rice. Plant Cell Physiol 46:699-715

Nishikura K (2001) A short primer on RNAi: RNA-directed RNA polymerase acts as a key catalyst. Cell 107:415-418

Sallaud C, Meynard D, van Boxtel J, Gay C, Bes M, Brizard JP, Larmande P, Ortega D, Raynal M, Portefaix M, Ouwerkerk PB, Rueb S, Delseny M, Guiderdoni E (2003) Highly efficient production and characterization of T-DNA plants for rice (Oryza sativa L.) functional genomics. Theor Appl Genet 106:1396-1408

Santos-Rosa M, Poutaraud A, Merdinoglu D, Mestre P (2008) Development of a transient expression system in grapevine via agro-infiltration. Plant Cell Rep 27:1053-1063

Shao F, Golstein C, Ade J, Stoutemyer M, Dixon JE, Innes RW (2003) Cleavage of Arabidopsis PBS1 by a bacterial type III effector. Science 301:1230-1233

Schöb H, Kunz C, Meins F (1997) Silencing of transgenes introduced into leaves by agroinfiltration: a simple, rapid method for investigating sequence requirements for gene silencing. Mol Gen Genet 256:581-585

Schweizer P, Pokorny J, Schulze-Lefert P, Dudler R (2000) Double-stranded RNA interferes with gene function at the single-cell level in cereals. Plant J 24:895-903

Sijen T, Fleenor J, Simmer F, Thijssen K, Parrish S, Timmons L, Plasterk R, Fire A (2001) On the role of RNA amplification in dsRNA-triggered gene silencing. Cell 107:465-476

Small I (2007) RNAi for revealing and engineering plant gene functions. Cur Opin Biotech 18:148-153

Tzfira T, Citovsky V (2002) Partners-in-infection: host proteins involved in the transformation of plant cells by Agrobacterium. Cell Biol 12:121-129

Vaistij FE, Jones L, Baulcombe DC (2002) Spreading of RNA targeting and DNA methylation in RNA silencing requires transcription of the target gene and a putative RNA-dependent RNA polymerase. Plant Cell 14:857-867

Vaquero C, Sack M, Chandler J, Drossard J, Schuster F, Monecke M, Schillberg S, Fischer R (1999) Transient expression of a tumor-specific single-chain fragment and a chimeric antibody in tobacco leaves. Proc Natl Acad Sci U S A 96:11128-11133

Van der Hoorn RA, Laurent F, Roth R, De Wit PJ (2000) Agroinfiltration is a versatile tool that facilitates comparative analyses of Avr9/Cf-9-induced and Avr4/Cf-4-induced necrosis. Mol Plant-Microbe Interact 13:439-446

Verdaguer B, de Kochko A, Fux Cl, Beachy RN, Fauquet C (1998) Functional organization of the cassava vein mosaic virus (CsVMV) promoter. Plant Mol Biol 37:1055-1067

Voinnet O (2001) RNA silencing as a plant immune system against viruses. Trends in Genetics 17:449-459

Voinnet O (2005a) Non-cell autonomous RNA silencing. FEBS Letters 579:5858-5871

Voinnet O (2005b) Induction and suppression of RNA silencing: insights from viral infections. Nat Rev Genet 6:206-220

Warthmann N, Chen H, Ossowski S, Weigel D, Hervé P (2008) Highly Specific Gene Silencing by Artificial miRNAs in Rice. PLoS One 3:e1829

Wroblewski T, Tomczak A, Michelmore R (2005) Optimization of Agrobacterium -mediated transient assays of gene expression in lettuce, tomato and Arabidopsis. Plant Biotech J 3:259-273

Wydro M, Kozubek E, Lehmann P (2006) Optimization of transient Agrobacteriummediated gene expression system in leaves of Nicotiana benthamiana. Acta Biochimica Polonica 53:289-298

Yang Y, Li R, Qi M (2000) In vivo analysis of plant promoters and transcription factors by agroinfiltration of tobacco leaves. Plant J 22:543-551

Zottini M, Barizza E, Costa A, Formentin E, Ruberti C, Carini F, Lo Schiavo F (2008) Agroinfiltration of grapevine leaves for fast transient assay of gene expression and for long-term production of stable transformed cells. Plant cell rep 27:845-854

\section{doi:10.1186/1939-8433-5-23}

Cite this article as: Andrieu et al:: An in planta, Agrobacterium-mediated transient gene expression method for inducing gene silencing in rice (Oryza sativa L.) leaves. Rice 2012 5:23. 